\title{
Temperature and solvent effects in the solubility of some pharmaceutical compounds: measurements and
}

\author{
modeling
}

Fátima L. Mota ${ }^{a}$, Aristides P. Carneiro ${ }^{a}$, António J. Queimada ${ }^{a}$, Simão P. Pinho ${ }^{b}$ and Eugénia A. Macedo, ${ }^{a, *}$

${ }^{a}$ LSRE/LCM - Laboratory of Separation and Reaction Engineering, Faculdade de Engenharia, Universidade do Porto, Rua Dr. Roberto Frias, 4200 - 465 Porto, Portugal

${ }^{b}$ LSRE/LCM - Laboratory of Separation and Reaction Engineering, Escola Superior de Tecnologia e Gestão, Instituto Politécnico de Bragança, Campus de Santa Apolónia, 5301857 Bragança, Portugal

*To whom correspondence should be addressed: eamacedo@fe.up.pt 


\section{Abstract}

In this work, pure solvent solubilities of drugs, such as paracetamol, allopurinol, furosemide and budesonide, measured in the temperature range $(298.2$ - 315.2) $\mathrm{K}$ are presented. The solvents under study were water, ethanol, acetone, ethyl acetate, carbon tetrachloride and n-hexane. Measurements were performed using the shake-flask method for generating the saturated solutions followed by compositional analysis by HPLC. Previous literature values on the solubilities of paracetamol were used to assess the experimental methodology employed in this work. No literature data was found for any of the other drugs studied in this assay. Melting properties of the pure drugs were also determined by differential scanning calorimetry (DSC) to provide a broader knowledge about the solubilization process and also for modeling purposes.

The solubility data as a function of temperature were used to determine the thermodynamic properties of dissolution like, Gibbs energy, enthalpy and entropy. Theoretical work was essentially focused on the evaluation of the Nonrandom Two-Liquid Segment Activity Coefficient (NRTL-SAC) model, which has been referred as a simple and practical thermodynamic framework for drug solubility estimation. A satisfactory agreement was found between experimental and calculated values: the absolute average deviation was $68 \%$ for the correlation in the organic solvents and $38 \%$ for the prediction in water, where the best results in prediction could be related to the selected solvents.

\section{Keywords: Drugs, Solubility, Measurement, Modeling, NRTL-SAC, Pure solvents.}




\section{Introduction}

One of the most challenging tasks of the pharmaceutical industry is to discover new therapies or to improve existing ones. Therefore, new drugs are evaluated everyday in the different steps of drug development. Many of these drug candidates are so hydrophobic that their effects in the organism, related to their bioavailability, are dependent on the techniques used by the pharmaceutical industry to make them more soluble (Blagden et al., 2007; Hu et al., 2004). These techniques include, among others, manipulation of solid state structures (polymorph changes and amorphous forms) (Nordström and Rasmuson, 2006), sonocrystallization (Manish et al., 2005), salt formation, solubilization in cosolvents and micellar solutions (Millard et al., 2002), complexation and the use of lypidic systems for the delivery of lipophilic drugs (Blagden et al., 2007). Pharmaceutical cocrystallization is a relatively new technology to improve solubilization (Basavoju et al., 2008; Blagden et al., 2007).

Solubility is, thus, a very important property for pharmaceutical product design because it affects the drug efficacy, its future development and formulation efforts, and also influences the pharmaco-kinetics, such as the release, transport and the degree of absorption in the organism. On the other hand, in the pharmaceutical industry, the majority of active pharmaceutical ingredients (API's) are isolated in the solid form via crystallization and so, solubility is important for the design of these processes. 
Solubility data involving new drugs are frequently not available in the literature. Although some thermodynamic models can be used to predict drug solubility, the availability of experimental data is still fundamental for an appropriate model development and evaluation. However, there are inherent complexities with experimental measurements: accuracy and reliability are difficult to achieve, and experiments are costly and time consuming. These difficulties can be related to substance purity, different solid structures, $\mathrm{pH}$, temperature control, and solubility measuring method. Stovall et al. (2005) and Bustamante et al. (2000) measured the solubility of ibuprofen in ethanol, methanol, 1pentanol and 1-octanol and the results between authors present differences in the range 9 $145 \%$, which stresses the need of a reliable experimental procedure and justifies why efficient methodologies to predict solubilities are a main research subject, with high value for drug design.

In spite of drugs being so complex molecules, with several functional groups (associating, hydrogen bonding), having different chemical structures or isomers, or even different solid structures, several methods to represent their solubility were reviewed (Pinho and Macedo, 2007). Some empirical correlations based on physicochemical properties like the octanol-water partition coefficient and the melting point were proposed (Huuskonen, 2001; Jorgensen and Duffy, 2002) but they are currently of little use. To calculate the partition coefficient several reliable methods are known, but that is not the case for the melting point. The so-called log-linear model (Millard et al., 2002) that uses the partition coefficient cannot predict solubility in systems that present maxima, and this is a reason 
why these empirical models should be replaced by more theoretically sound models such as the thermodynamic models regularly used for phase equilibria.

The Scatchard-Hildebrand concept of regular solutions (Prausnitz et al., 1999) has been one of the thermodynamic models most used in the pharmaceutical industry, and there are several modifications. According to this theory, the existence of a maximum in the solubility curve is observed when the solvent and solute solubility parameters are the same. This model was used for naproxen and sodium diclofenac in several solvents ranging from water, alkanes, alcohols, ketones, carboxylic acids, esters, ethers, amides, benzene and its derivatives (Bustamante et al., 1998b), proving to be suitable for determination of partialsolubility parameters. It was also used for paracetamol (Hojjati and Rohani, 2006a) but it underestimated the solubilities. With the correlative Hansen model (Bustamante et al., 1993), available experimental data are used to estimate solute solubility parameters. This model was successfully used to estimate solubility parameters using experimental data of sulfadiazine in some esters, toluene, benzene, dioxane, alcohols, water, amides, water and acetone, and sulfamethoxypyridazine in dioxane-water and ethyl acetate-ethanol mixtures (Bustamante et al., 1993). A drawback of this model is its very simple assumptions which lead to very limited practical use in the prediction of drug solubilities.

Some other activity coefficient models have also been used, but not all are practical because phase equilibrium data are frequently not available and the application of these models regularly requires the determination of binary interaction parameters from experimental data. The UNIFAC model (Fredenslund et al., 1975; Hojjati and Rohani, 2006a) is one interesting approach, used in the estimation of drug solubilities in pure and 
mixed solvents. This is a predictive group-contribution model, which requires only chemical structure information for the solutes and solvents. It was used in the prediction of the solubility of paracetamol in water-isopropanol mixtures (Hojjati and Rohani, 2006a), with overestimated solubilities being obtained. Additionally, when not all group parameters for the solute are available, group-contribution models cannot be used. Thus, they have to be obtained from structurally similar substances or from available experimental data. In this way, it often happens that the number of group parameters needed to be obtained is so high that the available database is insufficient to estimate all missing parameters.

Another methodology, proposed by Abildskov and O’Connell (2003; 2004; 2005), the reference solvent approach, is a simplified methodology that allows the prediction of differences in solubilities of sparingly soluble chemicals when the solvent is changed, based on a limited set of experimental data. This technique is useful because no pure-solute properties are needed. However, it requires the selection of a reference solvent. It was already tested with paracetamol in ethyl acetate/ethanol, and in sulfanilamide, phenobarbital and vinbarbital in water/ethanol mixtures (Abildskov and O'Connell, 2004), with estriol and ibuprofen (Abildskov and O'Connell, 2005) in ethanol/water solvent systems, and ephedrine, hydrocortisone, salicylic acid, niflumic acid, diuron and monuron in pure solvents (Abildskov and O'Connell, 2003). In general, this methodology cancels errors in pure-solute properties and eliminates many interaction parameters, showing a satisfactory accuracy and reliability. However, a strategy must be developed for solutes which are too much soluble and for those that form complexes or competitively interact 
with the solvent. The possible effect of solute structure modifications are also not taken into account.

The recent developments in computational chemistry yielded conductor-like screening model (COSMO) that are promising alternatives (Klamt and Eckert, 2000; Lin and Sandler, 2002; Mullins et al., 2008; Tung et al., 2008). The main characteristic of these models is the reduction of the molecular properties to a probability distribution of the screening charges for the solvated molecule in a perfect conductor, the sigma profile of the molecule. The advantage of this model is that no experimental data is required, needing only pre-calculated quantum chemical output. This model was applied for a set of drug compounds, namely lovastatin, simvastatin, rofecoxib and etoricoxib (Tung et al., 2008), with a reasonable prediction. Its implementation in the study of temperature influence of the solubilities of drug compounds in different solvents was also carried out (Žilnik et al., 2007) and even if the order of solubility magnitude is predicted, generally the results are poor.

The Nonrandom Two Liquid Segment Activity Coefficient (NRTL-SAC) model (Chen and Song, 2004; Chen and Crafts, 2006; Tung et al., 2008) is one recent and most successful model for the representation of drug solubility. It is based on the polymer NRTL model, a derivative of the original NRTL model of Renon and Prausnitz (1968), and has been widely applied to correlate and predict phase equilibria of highly nonideal systems, like those containing complex pharmaceutical organic electrolytes (Chen and Song, 2004; Chen and Crafts, 2006; Kokitkar and Plocharczyk, 2008). Chen and Song (2004) have shown that NRTL-SAC is a simple and practical framework to perform solubility 
calculations both in pure and mixed solvents based on a limited set of experimental data. The essence of this model consists in representing the molecules by conceptual segments. Accordingly, the experimental measurements to be considered for parameter regression must be carefully selected to cover the full range of segments. One of the advantages of NRTL-SAC is that it only requires this well-chosen data to fit a smaller number of parameters.

This work focus essentially poorly water soluble drugs such as budesonide, furosemide and allopurinol, whose water solubilities at the organism's temperature range from $20-500 \mathrm{mg} / \mathrm{L}$. Paracetamol was also used on the experimental measurements as it is one of the most studied drugs, allowing to validate the experimental procedure used in this work. The drugs selected have different functional groups and different molecular sizes, leading to different interactions with the solvents and, therefore, different properties. Also, the studied drugs have different therapeutic effects. Paracetamol is an active principle with analgesic/antipyretic action. Allopurinol is a xanthine oxidase inhibitor that decreases the uric acid production. Budesonide is a glucocorticoid steroid, used in the treatment of asthma and furosemide belongs to a family of benzoic-sulfonamide-furans, being a loop diuretic used in the treatment of congestive heart failure.

The selection of solvents used involved a careful choice to cover several types of surface interaction characteristics, namely, hydrophobic, hydrophilic and polar. Alkanes are hydrophobic compounds, ketones and esters are polar molecules with varying degrees of hydrophobic contents, alcohols are hybrids of hydrophobic and hydrophilic segments. In 
this way, n-hexane, carbon tetrachloride, acetone, ethyl acetate and ethanol were used as representative solvents, with distinctive surface interaction characteristics.

The main goal of this work is thus to extend the database on experimental solubility for a group of drugs and to evaluate the use of the NRTL-SAC model to correlate and predict the solubility of these compounds in the different solvents. In this way, solubilities were measured in a temperature range between 298.2 and $315.2 \mathrm{~K}$ in water, and at three temperatures $(298.2,310.2$, and $313.2 \mathrm{~K}$ ) in acetone, carbon tetrachloride, ethanol, ethyl acetate and n-hexane. Good agreement was obtained between experimental and modeling values: the absolute average deviation was $68 \%$ for the correlation, while it was $38 \%$ in the prediction.

\section{Material and Methods}

\subsection{Materials}

In all experiments, bi-distilled water $(\approx 2.5-3 \mu \mathrm{S} / \mathrm{cm})$ was used. Ethanol, acetone, ethyl acetate, carbon tetrachloride and n-hexane were of HPLC grade (99.8\% purity). Paracetamol (N-(4-hydroxyphenyl)acetamide, CAS No: 103-90-2, min. 99\% purity), allopurinol (3,5,7,8-tetrazabicyclo[4.3.0]nona-3,5,9-trien-2-one, CAS $N^{\mathrm{o}}$ : 315-30-0, min, 98\% purity), budesonide (16,17-(butylidenebis(oxy))-11,21-dihydroxy-,(11- $\beta, 16-\alpha)$ pregna-1,4-diene-3,20-dione, CAS No: 51333-22-3, min. 98\% purity) and furosemide (4chloro-2-(furan-2-ylmethylamino)-5-sulfamoylbenzoic acid, CAS No: 54-31-9, min, 99\% 
purity) were kindly provided by the Portuguese pharmaceutical company Bial. Their chemical structures are presented in Figure 1. All chemicals were used as received.

\subsection{Experimental Procedure}

\subsubsection{Solubility measurements}

All the solubility experiments were carried out using the analytical isothermal shake-flask method. The aqueous solubilities were measured at five different temperatures 298.2, 303.2, 310.2, 313.2 and $315.2 \mathrm{~K}$, while for the other solvents (ethanol, acetone, ethyl acetate, carbon tetrachloride and n-hexane) the solubilities were obtained at three temperatures, 298.2, 310.2 and 313.2 K. Saturated solutions were prepared mixing an excess of solid solute and $20 \mathrm{~mL}$ of solvent into a constant-temperature jacketed glass cells and stirring for 2 days. Temperature was maintained constant in two ways: i) using thermostatic water in the cell jackets and ii) placing the cells in an air bath. A circulating water bath (Grant LTC1, $\pm 0.1 \mathrm{~K}$ ) promoted the circulation of thermostatic water through the cell jackets; and the air bath, composed by an acrylic box, an electric fan and a resistance connected to a Fuji PXR-4 PID temperature controller $( \pm 0.1 \mathrm{~K})$ created a thermostatic environment around the cells, so that all the measuring procedure (stirring and sampling) was performed at the same target temperature. Temperature was monitored with 4-wire platinum resistance probes $(\mathrm{Pt}-100)( \pm 0.01 \mathrm{~K})$ placed in contact with solutions and connected to an Agilent 34970A data acquisition unit. This temperature measuring system was previously calibrated, presenting a maximum deviation of $0.06 \mathrm{~K}$ at $303.67 \mathrm{~K}$. Once the equilibrium was attained, the agitation was stopped and the solution was kept still for 1 
day allowing undissolved solid to settle. The stirring and settling times were determined by continuous sampling until no changes in composition were verified.

Samples of the saturated liquid phases were collected using plastic syringes coupled with polypropylene filters $(0.45 \mu \mathrm{m})$ previously placed at the air bath (in order they are at the same temperature as the samples). Quantitative analysis was then performed, for each drug, by comparison with a calibration curve using HPLC (VWR-Hitachi Lachrom Elite) with Uv-Vis detection using a reversed-phase C18 HPLC column (Merck Purospher® star RP18e). Each solubility value is an average of at least three different measurements, which are presented in Table 1, simultaneously with the respective standard deviations.

\subsubsection{DSC measurements}

Melting data of the pure compounds were obtained by differential scanning calorimetry (DSC) (Netzsch 200 F3 Maia) to provide a broader understanding of the solubilization process, as well as for modeling purposes. Aluminium crucibles were used to hermetically seal samples (4-6 mg) of solid and an empty crucible was used as reference. The heating was promoted under a stream of nitrogen. The first run was done in a larger temperature range with a heating rate of $10 \mathrm{~K} / \mathrm{min}$ and then, several runs (at least 3) were done at $1 \mathrm{~K} / \mathrm{min}$ around the expected melting temperature. The accuracy of the equipment was ascertained running an indium standard. Each value is an average of at least three runs, which are presented in Table 2, together with the number of measurements and the corresponding standard deviations. 


\subsection{Thermodynamic modeling}

The solubility of a solid solute $s$ in a liquid solvent is given by the following simplified expression, where it is assumed that the solid phase is pure and that the triple point temperature can be replaced by the melting temperature (Prausnitz et al., 1999):

$$
\ln x_{s}=-\frac{\Delta_{f u s} H}{R}\left(\frac{1}{T}-\frac{1}{T_{m}}\right)+\frac{\Delta C_{p}}{R}\left[\frac{T_{m}}{T}-1-\ln \left(\frac{T_{m}}{T}\right)\right]-\ln \gamma_{s}
$$

where $x_{s}$ is the mole fraction of the solute, $\Delta_{f u s} H$ is the solute enthalpy of fusion, $R$ is the ideal gas constant, $T$ the absolute temperature, $T_{m}$ the solute melting temperature, $\Delta C_{p}$ is the difference between the molar heat capacity of solute liquid and solid phases (considered to be temperature independent) and $\gamma_{s}$ is the solute activity coefficient calculated using a thermodynamic model.

The NRTL-SAC model (Chen and Song, 2004) is a modification of the original NRTL where the activity coefficient of component $\mathrm{I}\left(\gamma_{I}\right)$ is obtained from two contributions: a combinatorial term $\left(\gamma_{I}^{C}\right)$ obtained from the Flory-Huggins approximation for the combinatorial entropy of mixing and a residual term $\left(\gamma_{I}^{R}\right)$ set equal to the sum of the local composition $(l c)$ interaction contribution for each segment.

$$
\ln \gamma_{I}=\ln \gamma_{I}^{C}+\ln \gamma_{I}^{R}
$$


The combinatorial term is given by:

$$
\ln \gamma_{I}^{C}=\ln \frac{\phi_{I}}{x_{I}}+1-r_{I} \sum_{J} \frac{\phi_{J}}{r_{J}}
$$

with

$$
\begin{gathered}
r_{I}=\sum_{i} r_{i, I} \\
\phi_{I}=\frac{r_{I} x_{I}}{\sum_{J} r_{J} x_{J}}
\end{gathered}
$$

where $x_{J}$ is the mole fraction of component $J, r_{m, I}$ is the number of segment species $m$ contained in component $I, r_{I}$ is the total segment number of component $I$ and $\phi_{I}$ is the segment mole fraction of component $I$.

The residual term,

$$
\ln \gamma_{I}^{R}=\ln \gamma_{I}^{l c}=\sum_{m} r_{m, I}\left(\ln \Gamma_{m}^{l c}-\ln \Gamma_{m}^{l c, I}\right)
$$

where the activity coefficient of segment species $m\left(\Gamma_{m}^{l c}\right)$ is given by:

$$
\ln \Gamma_{m}^{l c}=\frac{\sum_{j} x_{j} G_{j m} \tau_{j m}}{\sum_{k} x_{k} G_{k m}}+\sum_{m^{\prime}} \frac{x_{m^{\prime}} G_{m m^{\prime}}}{\sum_{k} x_{k} G_{k m^{\prime}}}\left(\tau_{m m^{\prime}}-\frac{\sum_{j} x_{j} G_{j m^{\prime}} \tau_{j m^{\prime}}}{\sum_{k} x_{k} G_{k m^{\prime}}}\right)
$$


and the activity coefficient of segment species $m$ contained only in component $I\left(\Gamma_{m}^{l c, I}\right)$ is expressed by:

$$
\ln \Gamma_{m}^{l c, I}=\frac{\sum_{j} x_{j, I} G_{j m} \tau_{j m}}{\sum_{k} x_{k, I} G_{k m}}+\sum_{m^{\prime}} \frac{x_{m^{\prime}, I} G_{m m^{\prime}}}{\sum_{k} x_{k, I} G_{k m^{\prime}}}\left(\tau_{m m^{\prime}}-\frac{\sum_{j} x_{j, I} G_{j m^{\prime}} \tau_{j m^{\prime}}}{\sum_{k} x_{k, I} G_{k m^{\prime}}}\right)
$$

$I$ and $J$ are component indexes, $i, j, k, m$ and $m$ ' are segment based species indexes, $x_{j}$ is the segment-based mole fraction of segment species $j, x_{j, I}$ is the segment-based mole fraction of segment species $j$ contained only in component $I$, and can be calculated as follows:

$$
\begin{gathered}
x_{j}=\frac{\sum_{J} x_{J} r_{j, J}}{\sum_{I} \sum_{i} x_{I} r_{i, I}} \\
x_{j, I}=\frac{r_{j, I}}{\sum_{i} r_{i, I}}
\end{gathered}
$$

$G_{i j}$ and $\tau_{i j}$ are local binary quantities related to each other by the NRTL nonrandomness parameter $\alpha$ :

$$
G_{i j}=\exp \left(-\alpha_{i j} \tau_{i j}\right)
$$

To account for several interactions, predefined conceptual segments were proposed, along with their corresponding segment-segment binary parameters, and this is what makes 
the difference between this model and the group-contribution approaches which build molecules from a large set of predefined functional groups based on chemical structure. Each compound in the system is represented by predefined segments, one hydrophobic $(X)$, one polar-attractive $(Y-)$, one polar-repulsive $(Y+)$ and one hydrophilic $(Z)$. The hydrophobic segment represents the molecular surface area unlike to form hydrogen bonds; the hydrophilic represents the area with interactions characteristic of a hydrogen-bond donor or acceptor; the polar represent that area with interactions characteristic of an electron donor or acceptor. In the set of model equations, these segments are represented by the segment number $\left(r_{m, I}\right)$ : which has a specific value for each component. For most of the solvents used in the pharmaceutical industry, these parameters are already available (Chen and Crafts, 2006) and Table 3(a) presents the parameters for the solvents used in this work. The segment numbers of each solute are the model parameters and in this case they are four. The chosen values for the NRTL binary interaction parameters, $\alpha$ and $\tau$, for the conceptual segments, which appear only in the residual term, are those reported by Chen and Song (2004) and they are shown in Table 3(b). The parameters presented in Tables 3a and $3 \mathrm{~b}$ were determined by regression of vapor-liquid and liquid-liquid equilibrium data. With NRTL-SAC, available experimental data are used to estimate the solute model parameters, and afterwards the model can be applied to predict solute solubility for other solvent systems (Chen and Song, 2004).

The melting properties have also an important role in the solubilization of solids in liquids and are fundamental for modeling purposes. If they cannot be experimentally measured, they can be estimated using a third-order group-contribution method proposed 
by Marrero and Gani (2001), able to distinguish between different types of isomers and already tested with success for complex chemicals (Mota et al., 2008).

\section{Results and Discussion}

The measured solubilities of paracetamol, allopurinol, budesonide and furosemide, as a function of temperature were presented in Table 1. The solubility follows the expected increasing trend with temperature. Paracetamol and allopurinol are the most soluble in water, in the range of $0.2-38 \mathrm{~g} / \mathrm{L}$, while budesonide and furosemide are the less soluble (less than $55 \mathrm{mg} / \mathrm{L}$ ).

Solubilities in the pure organic solvents are also listed in Table 1 while Table 1(a) shows a solubility ranking. Solubilities are higher in the binary systems paracetamol/ethanol and paracetamol/acetone with the lowest being found for furosemide/carbon tetrachloride and furosemide/n-hexane. This means that, for example, ethanol and acetone are appropriate solvents to separate and purify paracetamol from solutions. n-Hexane is among the studied solvents the worst to solubilize all of these drugs (except for budesonide, where water takes its place), and the best solvents are ethanol and acetone.

With the exception of paracetamol, among the studied drugs no solubility data are available in the literature. The results obtained for paracetamol were compared with data published by other authors, which are all presented and discriminated in Figure 2. In 
general, the values are in good agreement. The aqueous solubility measured deviate in average $14 \%$ with respect to the data presented by Bustamante et al. (1998a), $11 \%$ to that presented by Granberg and Rasmuson (2000) and 17\% to Hojatti and Rohani (2006b) . For water, at the lowest temperatures, the values are in good agreement. In this work, the measured value was $13.05 \mathrm{~g} / \mathrm{L}( \pm 0.39)$ at $293.2 \mathrm{~K}$ which deviates less than $9 \%$ from the value reported by the other authors. Near $310.2 \mathrm{~K}$, the aqueous solubilities from this work are higher than those reported in the literature (maximum deviation in the order of $23 \%$ ). Still, as mentioned before, deviations of this magnitude are typically found when comparing solubility data from different authors, and the consistency found in solubility values measured support this observation.

The measured data for acetone are in very good agreement with those presented by Granberg and Rasmuson (2000), deviating in average 1\%. For ethanol, $1 \%$ is the average deviation with respect to the data presented by Granberg and Rasmuson (1999), 11\% to data presented by Jimenez and Martinez (2006) and 19\% to those presented by Romero et al. (1996). For n-hexane, the measured data deviate in average $14 \%$ when compared with the data presented by Perlovich et al. (2006).

Furosemide and allopurinol decompose at melting $(534.3 \mathrm{~K}$ and $653.5 \mathrm{~K}$, respectively), and because of this, their fusion enthalpies could not be measured. Melting data was found for paracetamol (BYU DIPPR 801 Thermophysical Properties Database, 1998; Chen and Crafts, 2006; NIST Chemistry Webbook, 2007) with melting point deviating less than $1 \%$ and enthalpy of fusion less than $6 \%$. Melting temperatures of $633 \mathrm{~K}$ and $479 \mathrm{~K}$ were found (Jain et al., 2008) for allopurinol and furosemide respectively, that 
compare well with the values measured in this work (deviations of 3 and $12 \%$, respectively). The values measured in this work are in close agreement with those reported by Bial: melting temperatures of $512.2 \mathrm{~K}$ and $443.2 \mathrm{~K}$ for budesonide and paracetamol, respectively.

For paracetamol, budesonide and furosemide the calculated melting temperatures are in good agreement with the experimental data (deviations below 12\%) while the calculated fusion enthalpies present higher deviations (15\% for paracetamol and 64\% for budesonide).

DSC analysis of the pure drugs were performed in the temperature range from 273 up to some $\mathrm{K}$ above the melting temperature, and single and sharp peaks were found, indicating that a single crystal structure was being studied.

The solubility data of the drugs under study was plotted as function of the temperature to calculate the thermodynamic properties of dissolution, molar Gibbs energy $\left(\Delta_{\text {sol }} G\right)$, enthalpy $\left(\Delta_{\text {sol }} H\right)$ and entropy $\left(\Delta_{\text {sol }} S\right)$.

Linear solubility-temperature plots are often considered desirable, because they can be interpolated and extrapolated quite accurately, can be treated by the usual statistics of linear regression and can provide thermodynamic data for the dissolution process. The original van't Hoff plot of $\ln$ (solubility) against 1/T are undoubtedly most helpfully, however to express the temperature dependence of solubility for nearly ideal solid-liquid systems a more theoretically-based approach is adopted. It starts from the Gibbs-Helmholtz equation and the definition of the solubility product in terms of the molar Gibbs energy of dissolution, from where the following equations can be obtained (Adkins, 1983): 


$$
\begin{gathered}
\Delta_{s o l} H=R T^{2}\left(\frac{d \ln x}{d T}\right)_{P} \\
\Delta_{s o l} G=-R T \ln (x)_{P}
\end{gathered}
$$

where the molar enthalpy of dissolution is the difference between the partial molar enthalpy of the compound in the solution and the pure molar enthalpy at temperature T, $x$ is the solute mole fraction solubility, and $P$ is the pressure. The values of the enthalpy of dissolution can be regarded as a reflection of the nature of intermolecular interactions.

The entropic change for the dissolution process $\left(\Delta_{s o l} S\right)$ is obtained from the respective enthalpies and Gibbs energies:

$$
\Delta_{s o l} S=\frac{\Delta_{s o l} H-\Delta_{s o l} G}{T}
$$

Table 4 summarizes the thermodynamic functions for the dissolution process in pure solvents. Looking at the thermodynamic properties in water, it is possible to observe that budesonide presents the smallest enthalpy of dissolution. This would favor its dissolution relatively to the other drugs, but because of a very high (and negative) entropy of dissolution it is hindered as involves a considerable ordering of the water molecules. In fact, budesonide being such a large molecule requires a considerable amount of water hydrogen bonds to be broken in order to be solubilized. The same is somehow found for 
furosemide, another large molecule, where the entropy of dissolution is very close to zero or slightly negative at the lowest temperatures. Comparing paracetamol and allopurinol it can be seen that paracetamol has a smaller enthalpy of dissolution and then it is more soluble.

Except for paracetamol, no literature values were found for comparison. Bustamante et al. (1998a) reported a $\Delta_{s o l} H$ of $22.46 \mathrm{~kJ} / \mathrm{mol}$ close to $303 \mathrm{~K}$, while in this work it was found $35.61 \mathrm{~kJ} / \mathrm{mol}$. This value was calculated by a method slightly different from that used in this work: in the temperature range from 278 to $343 \mathrm{~K}$ they used a nonlinear tendency, while here a linear fit was adopted between 298.2 and $315.2 \mathrm{~K}$.

Looking at the organic solvents (Table 4), paracetamol in carbon tetrachloride has a very favorable entropic contribution but a strong enthalpic contribution, leading to smaller solubility. Budesonide has very similar enthalpic contributions in acetone, carbon tetrachloride and ethanol; in water and n-hexane where the enthalpic contributions are low, but there are negative entropies, the solubilities are considerably lower. Furosemide has entropic effects determining its smaller dissolution in $\mathrm{n}$-hexane while in carbon tetrachloride the enthalpic effects are more relevant. Allopurinol also has negative entropies determining its low solubilities in carbon tetrachloride and ethyl acetate. All the enthalpies of solution are positive, meaning that the dissolution processes are endothermic.

Like previously mentioned, to calculate solubilities it is required an activity coefficient model, melting properties, and the difference of the heat capacities between the hypothetical liquid and solid phases. Limited data are available for $\Delta C_{p}$. Only for paracetamol a value was found: $32.13 \mathrm{~J} / \mathrm{mol} . \mathrm{K}$ at $298.2 \mathrm{~K}$ (BYU DIPPR 801 
Thermophysical Properties Database, 1998). For the other compounds, the values had to be estimated. For the liquid heat capacity, a three-level group-contribution method first proposed by Marrero and Gani (2001) and extended to take into account temperature effects was used (Kolská et al., 2008). For the solid heat capacity, a correlation based on molecular structure was selected (Goodman et al., 2004). The following estimated values were found at $298.2 \mathrm{~K}: 121.32 \mathrm{~J} / \mathrm{mol} . \mathrm{K}$ for paracetamol, $188.30 \mathrm{~J} / \mathrm{mol} . \mathrm{K}$ for furosemide, $162.01 \mathrm{~J} / \mathrm{mol} . \mathrm{K}$ for allopurinol and $104.34 \mathrm{~J} / \mathrm{mol} . \mathrm{K}$ for budesonide. In spite of, the estimated value for paracetamol being higher $(73 \%)$ than that found in literature, the estimated values will be used as reference values. Frequently, in solubility calculations, the $\Delta C_{p}$ is ignored, specially when compounds have high melting points (Mishra and Yalkowsky, 1992). This is the case of allopurinol (melting point $653.5 \mathrm{~K}$ ), where the $\Delta C_{p}$ term will not be considered.

Concerning the optimization procedure, for each system the parameters were estimated minimizing the following objective function:

$$
F=\sum_{i}\left(\frac{\ln x_{s}^{\exp }-\ln x_{s}^{c a l c}}{\ln x_{s}^{\exp }}\right)^{2}
$$

where $x_{s}$ is the solute solubility in mole fraction and the superscripts exp and calc refer to experimental and calculated solubility, respectively.

The solute model parameters were regressed using the solubility data in pure solvents presented in Table 1, the melting data shown in Table 2, the $\Delta C_{p}$ values above 
mentioned and the solvent parameters published by Chen and Crafts (2006) (Tables 3). The aqueous solubilities will be predicted and because of that water was not included in the regression. Table 5 presents the conceptual segment numbers for each drug under study together with the absolute average deviation (AAD). Chen and Crafts (2006) have published different parameters for paracetamol, but this difference can be related to their different melting temperature and enthalpy of fusion, and not considering the $\Delta C_{p}$ term in the estimation of the parameters. Also, these authors used a more extensive database. In this work, the parameters were regressed based in five solvents only.

The correlation results lead to the conclusion that NRTL-SAC is an adequate method, with a maximum deviation of $89 \%$ for budesonide. For this compound all solubilities are low and deviations higher than $80 \%$ were found for all solvents. Ethanol was the solvent for which lower deviations were found, in the order of $53 \%$, while nhexane, carbon tetrachloride and ethyl acetate were those with higher deviations, in the order of 82,75 and $65 \%$, respectively. The most soluble compounds, allopurinol and paracetamol, showed much better correlation results.

Once the solute model parameters are obtained, the model can be used to predict the solubility of the same solute in different solvents. Figure 3 shows the prediction of the aqueous solubilities of these compounds as a function of temperature. In general, NRTLSAC provides a good prediction, with a maximum deviation of $70 \%$ for allopurinol, which for a predictive result of aqueous solubility can still be considered a good estimate, as solubilities of such complex organic compounds in water are frequently difficult to predict. Surprisingly, allopurinol was the drug that showed lower deviation during correlation. 
However, the correlation was based in five solvents where ethanol is the only one presenting the unique kind of segment (hydrophilic) present in water. Moreover, the allopurinol hydrophilic segment parameter was fixed to zero because the regressed value was too low and some correlation and prediction tests were made fixing it at zero and the results did not vary. It can also be observed that the prediction results are better than correlation, what generally not happen. This can be related to the fact that solutes present a broader and more complex kind of interactions with the solvents selected for correlation than with water.

The same model parameters can also be used to predict the solubility in mixed solvents. For paracetamol, Granberg and Rasmuson (2000) reported solubility data in mixtures of water and acetone; Romero et al. (1996) reported data for ethanol-water and ethanol-ethyl acetate binary solvents. In the case of the binary mixture ethanol-water, a solubility peak for higher water weight fractions was observed, where the predictions are not in good agreement with the experimental data. Even though, the average deviations for water weight fractions between 0.0 and 0.7 are $59 \%$. In the case of the binary mixture acetone-water, although this is an extremely non-ideal system, the model predictions are in good agreement with the experimental data and the average deviations for water weight fractions between 0.0 and 0.5 are $26 \%$, similar to what was found for the mixed solvent system ethyl acetate/ ethanol, presented in Figure 4. In this figure a comparison between the results obtained with the paracetamol parameters estimated in this work and those proposed by Chen and Crafts (2006) is also shown. The average deviations obtained with the parameters reported by those authors are $19 \%$, lower than those using the parameters 
calculated in this work (46\%), but Chen and Crafts (2006) used five adjustable parameters, a much larger set of solvents for parameter estimation, and did not took into account the melting properties and the difference in the thermal heat capacity. It can also be seen that for pure ethanol, the calculated solubility value is very good (absolute deviation of 18\%), but as long as the ethyl acetate weight fraction increases the prediction gets worse. When it reaches pure ethyl acetate, the correlated solubility value presents a deviation of $69 \%$. As referred before, ethyl acetate was one of the solvents where the correlation was worse, and that certainly influences the quality of predictions. Even though, if the ideal solubilities were calculated, the absolute average deviation would be $75 \%$, indicating that considering the non-ideality of the real mixture, a considerable improvement is achieved.

These results showed that NRTL-SAC is a good correlative and predictive model for single and binary solvents system with the solutes under study. Even if high deviations may be found, the results, and particularly the predictions, were very satisfactory, showing that the regressed solute parameters are very useful for drug solubility predictions in solvents not studied experimentally.

\section{Conclusions}


In this work, aqueous solubility data were measured for some drug compounds (paracetamol, budesonide, allopurinol and furosemide) in the temperature range between 298.2 K and 315.2 K. For all compounds under study, the solubility follows the general increasing trend with temperature. Solubility data were also measured in a set of organic solvents (ethanol, acetone, ethyl acetate, carbon tetrachloride and n-hexane) at 298.2, 310.2 and $313.2 \mathrm{~K}$. The standard shake-flask method coupled with HPLC analysis was used to generate and analyse the saturated solutions. Besides solubility data, melting temperatures and enthalpies of fusion were also determined, providing a broader knowledge about the solubilization process of these molecules, and enabling also modeling purposes. The enthalpy, Gibbs energy and entropy of dissolution were determined allowing a better understanding of the solubilization process.

The NRTL-SAC model was successfully applied to estimate drug solubilities. The solubility data in pure organic solvents were used to regress the solute model parameters, which were used after for the prediction of the solubility of these compounds in water and in mixed solvent systems. This model showed to be an appropriate tool to represent and predict the solubility of these molecules.

\section{Acknowledgements}

The authors are grateful for the support provided by Fundação para a Ciência e a Tecnologia (FCT, Lisboa, Portugal) and by LSRE financing by FEDER/POCI/2010 and to REEQ/1164/EQU/2005. Fátima L. Mota acknowledges her FCT Ph.D. scholarship 
SFRH/BD/32372/2006 and A. J. Queimada acknowledges financial support from POCI/N010/2006. The authors are also very grateful to Bial for kindly providing the drugs used in this work.

\section{References}

Abildskov, J., O'Connell, J.P., 2003. Predicting the solubilities of complex chemicals I. Solutes in different solvents. Ind. Eng. Chem. Res. 42, 5622-5634.

Abildskov, J., O'Connell, J.P., 2004. Prediction of solubilities of complex medium-sized chemicals. II. Solutes in mixed solvents. Mol. Sim. 30, 367-378.

Abildskov, J., O'Connell, J.P., 2005. Thermodynamic method for obtaining the solubilities of complex medium-sized chemicals in pure and mixed solvents. Fluid Phase Equilib. 228, 395-400.

Adkins, C.J., 1983. Equilibrium Thermodynamics. Cambridge University Press, Cambridge.

Basavoju, S., Bostrom, D., Velaga, S.P., 2008. Indomethacin-saccharin cocrystal: Design, synthesis and preliminary pharmaceutical characterization. Pharm. Res. 25, 530-541.

Blagden, N., de Matas, M., Gavan, P.T., York, P., 2007. Crystal engineering of active pharmaceutical ingredients to improve solubility and dissolution rates. Adv. Drug Del. Rev. $59,617-630$.

Bustamante, P., Martin, A., Gonzalezguisandez, M.A., 1993. Partial solubility parameters and solvatochromic parameters for predicting the solubility of single and multiple-drugs in individual solvents. J. Pharm. Sci. 82, 635-640. 
Bustamante, P., Romero, S., Peña, A., Escalera, B., Reillo, A., 1998a. Enthalpy-entropy compensation for the solubility of drugs in solvent mixtures: Paracetamol, acetanilide, and nalidixic acid in dioxane-water. J. Pharm. Sci. 87, 1590-1596.

Bustamante, P., Peña, A., Barra, J., 1998b. Partial-solubility parameters of naproxen and sodium diclofenac. J. Pharm. Pharmacol. 50, 975-982.

Bustamante, P., Peña, M.A., Barra, J., 2000. The modified extended Hansen method to determine partial solubility parameters of drugs containing a single hydrogen bonding group and their sodium derivatives: benzoic acid/Na and ibuprofen/Na. Int. J. Pharm. 194, $117-124$.

BYU DIPPR 801 Thermophysical Properties Database. AIChE 1998.

Chen, C.C., Song, Y.H., 2004. Solubility modeling with a nonrandom two-liquid segment activity coefficient model. Ind. Eng. Chem. Res. 43, 8354-8362.

Chen, C.C., Crafts, P.A., 2006. Correlation and prediction of drug molecule solubility in mixed solvent systems with the nonrandom two-liquid segment activity coefficient (NRTLSAC) model. Ind. Eng. Chem. Res. 45, 4816-4824.

Fredenslund, A., Jones, R.L., Prausnitz, J.M., 1975. Group-Contribution Estimation of Activity-Coefficients in Nonideal Liquid-Mixtures. AICHE J. 21, 1086-1099.

Goodman, B.T., Wilding, W.V., Oscarson, J.L., Rowley, R.L., 2004. Use of the DIPPR database for development of quantitative structure-property relationship correlations: Heat capacity of solid organic compounds. J. Chem. Eng. Data 49, 24-31.

Granberg, R.A., Rasmuson, A.C., 1999. Solubility of paracetamol in pure solvents. J. Chem. Eng. Data 44, 1391-1395. 
Granberg, R.A., Rasmuson, A.C., 2000. Solubility of paracetamol in binary and ternary mixtures of water plus acetone plus toluene. J. Chem. Eng. Data 45, 478-483.

Hojjati, H., Rohani, S., 2006a. Measurement and prediction of solubility of paracetamol in water-isopropanol solution. Part 2. Prediction. Org. Process Res. Dev. 10, 1110-1118.

Hojjati, H., Rohani, S., 2006b. Measurement and prediction of solubility of paracetamol in water-isopropanol solution. Part 1. Measurement and data analysis. Org. Process Res. Dev. 10, 1101-1109.

Hu, J.H., Johnston, K.P., Williams, R.O., 2004. Nanoparticle engineering processes for enhancing the dissolution rates of poorly water soluble drugs. Drug Dev. Ind. Pharm. 30, 233-245.

Huuskonen, J., 2001. Estimation of aqueous solubility in drug design. Combinatorial Chem. High Throughput Screening 4, 311-316.

Jain, P., Sepassi, K., Yalkowsky, S.H., 2008. Comparison of aqueous solubility estimation from AQUAFAC and the GSE. Int. J. Pharm. 360, 122-147.

Jimenez, J.A., Martinez, F., 2006. Temperature dependence of the solubility of acetaminophen in propylene glycol plus ethanol mixtures. J. Solution Chem. 35, 335-352. Jorgensen, W.L., Duffy, E.M., 2002. Prediction of drug solubility from structure. Adv. Drug Del. Rev. 54, 355-366.

Klamt, A., Eckert, F., 2000. COSMO-RS: a novel and efficient method for the a priori prediction of thermophysical data of liquids. Fluid Phase Equilib. 172, 43-72.

Kokitkar, P.B., Plocharczyk, E., 2008. Modeling drug molecule solubility to identify optimal solvent systems for crystallization. Org. Process Res. Dev. 12, 249-256. 
Kolská, Z., Kukal, J., Zábranský, M., Růžička, V., 2008. Estimation of the heat capacity of organic liquids as a function of temperature by a three-level group contribution method. Ind. Eng. Chem. Res. 47, 2075-2085.

Lin, S.T., Sandler, S.I., 2002. A priori phase equilibrium prediction from a segment contribution solvation model. Ind. Eng. Chem. Res. 41, 899-913.

Manish, M., Harshal, J., Anant, P., 2005. Melt sonocrystallization of ibuprofen: Effect on crystal properties. Eur. J. Pharm. Sci. 25, 41-48.

Marrero, J., Gani, R., 2001. Group-contribution based estimation of pure component properties. Fluid Phase Equilib. 183, 183-208.

Millard, J.W., Alvarez-Nunez, F.A., Yalkowsky, S.H., 2002. Solubilization by cosolvents Establishing useful constants for the log-linear model. Int. J. Pharm. 245, 153-166.

Mishra, D.S., Yalkowsky, S.H., 1992. Ideal solubility of a solid solute - Effect of heatcapacity assumptions. Pharm. Res. 9, 958-959.

Mota, F.L., Queimada, A.J., Pinho, S.P., Macedo, E.A., 2008. Aqueous solubility of some natural phenolic compounds. Ind. Eng. Chem. Res. 47, 5182-5189.

Mullins, E., Liu, Y.A., Ghaderi, A., Fast, S.D., 2008. Sigma profile database for predicting solid solubility in pure and mixed solvent mixtures for organic pharmacological compounds with COSMO-based thermodynamic methods. Ind. Eng. Chem. Res. 47, 1707-1725.

NIST Chemistry Webbook, 2007. http://webbook.nist.gov/chemistry/.

Nordström, F.L., Rasmuson, A.C., 2006. Polymorphism and thermodynamics of mhydroxybenzoic acid. Eur. J. Pharm. Sci. 28, 377-384.

Perlovich, G.L., Volkova, T.V., Bauer-Brandl, A., 2006. Towards an understanding of the molecular mechanism of solvation of drug molecules: A thermodynamic approach by 
crystal lattice energy, sublimation, and solubility exemplified by paracetamol, acetanilide, and phenacetin. J. Pharm. Sci. 95, 2158-2169.

Pinho, S.P., Macedo, E.A., 2007. Solubility in Food, Pharmaceutical and Cosmetic Industries. In: Letcher, T.M. (Ed.), Developments and Applications in Solubility. Royal Society of Chemistry, Cambridge, pp. 309-326.

Prausnitz, J.M., Lichtenthaler, R.N., Azevedo, E.G., 1999. Molecular Thermodynamics of Fluid-Phase Equilibria. Prentice Hall PTR, New Jersey.

Renon, H., Prausnit.Jm, 1968. Local compositions in thermodynamic excess functions for liquid mixtures. AICHE J. 14, 135-\&.

Romero, S., Reillo, A., Escalera, B., Bustamante, P., 1996. The behavior of paracetamol in mixtures of amphiprotic and amphiprotic-aprotic solvents. Relationship of solubility curves to specific and nonspecific interactions. Chem. Pharm. Bull. (Tokyo) 44, 1061-1064.

Stovall, D.M., Givens, C., Keown, S., Hoover, K.R., Rodriguez, E., Acree, W.E., Abraham, M.H., 2005. Solubility of crystalline nonelectrolyte solutes in organic solvents: Mathematical correlation of ibuprofen solubilities with the Abraham solvation parameter model. Phys. Chem. Liq. 43, 261-268.

Tung, H.H., Tabora, J., Variankaval, N., Bakken, D., Chen, C.C., 2008. Prediction of pharmaceutical solubility via NRTL-SAC and COSMO-SAC. J. Pharm. Sci. 97, 18131820.

Žilnik, L.F., Jazbinšek, A., Hvala, A., Vrečer, F., Klamt, A., 2007. Solubility of sodium diclofenac in different solvents. Fluid Phase Equilib. 261, 140-145. 
Table 1 - Experimental solubilities (s) of the studied drugs.

\begin{tabular}{|c|c|c|c|c|}
\hline \multirow{2}{*}{$\mathbf{T}(\mathbf{K})$} & \multicolumn{4}{|c|}{$s(g / L)$} \\
\hline & paracetamol & allopurinol & budesonide & furosemide \\
\hline \multicolumn{5}{|c|}{ water } \\
\hline 315 & $37.51 \pm 0.81$ & $0.58 \pm 0.01$ & $(25.76 \pm 0.14) \times 10^{-3}$ & $(55.12 \pm 0.34) \times 10^{-3}$ \\
\hline 313 & $32.37 \pm 0.59$ & $0.55 \pm 0.01$ & $(24.46 \pm 0.62) \times 10^{-3}$ & $(45.48 \pm 0.43) \times 10^{-3}$ \\
\hline 310 & $27.33 \pm 2.97$ & $0.42 \pm 0.02$ & $(24.13 \pm 0.20) \times 10^{-3}$ & $(35.22 \pm 0.20) \times 10^{-3}$ \\
\hline 303 & $20.71 \pm 0.68$ & $0.28 \pm 0.01$ & $(20.90 \pm 0.31) \times 10^{-3}$ & $(30.10 \pm 0.05) \times 10^{-3}$ \\
\hline 298 & $16.66 \pm 1.12$ & $0.20 \pm 0.02$ & $(19.04 \pm 0.33) \times 10^{-3}$ & $(25.32 \pm 1.03) \times 10^{-3}$ \\
\hline \multicolumn{5}{|c|}{ acetone: } \\
\hline 313 & $140.11 \pm 5.85$ & $2.49 \pm 0.02$ & $8.45 \pm 0.39$ & $4.36 \pm 0.05$ \\
\hline 310 & $131.47 \pm 2.73$ & $1.33 \pm 0.05$ & $6.96 \pm 0.42$ & $3.03 \pm 0.10$ \\
\hline 298 & $98.44 \pm 3.92$ & $0.66 \pm 0.03$ & $3.98 \pm 0.18$ & $2.19 \pm 0.12$ \\
\hline \multicolumn{5}{|c|}{ carbon tetrachloride } \\
\hline 313 & $(21.97 \pm 1.28) \times 10^{-3}$ & $(101.53 \pm 0.45) \times 10^{-3}$ & $(817.22 \pm 11.53) \times 10^{-3}$ & $(1.57 \pm 0.18) \times 10^{-3}$ \\
\hline 310 & $(16.68 \pm 1.24) \times 10^{-3}$ & $(82.59 \pm 0.38) \times 10^{-3}$ & $(612.19 \pm 6.28) \times 10^{-3}$ & $(0.93 \pm 0.05) \times 10^{-3}$ \\
\hline 298 & $(4.64 \pm 0.14) \times 10^{-3}$ & $(66.65 \pm 0.41) \times 10^{-3}$ & $(412.70 \pm 7.86) \times 10^{-3}$ & $(0.28 \pm 0.01) \times 10^{-3}$ \\
\hline \multicolumn{5}{|c|}{ ethanol } \\
\hline 313 & $242.69 \pm 4.27$ & $(152.63 \pm 7.30) \times 10^{-3}$ & $31.37 \pm 1.84$ & $16.03 \pm 0.42$ \\
\hline 310 & $224.35 \pm 3.79$ & $(92.17 \pm 5.36) \times 10^{-3}$ & $23.99 \pm 0.99$ & $6.87 \pm 0.51$ \\
\hline 298 & $188.09 \pm 2.34$ & $(36.10 \pm 4.33) \times 10^{-3}$ & $14.94 \pm 1.16$ & $2.25 \pm 0.16$ \\
\hline \multicolumn{5}{|c|}{ ethyl acetate } \\
\hline 313 & $(31.87 \pm 1.43) \times 10^{-3}$ & $(210.36 \pm 0.67) \times 10^{-3}$ & $8.38 \pm 0.06$ & $10.68 \pm 1.14$ \\
\hline 310 & $(23.64 \pm 0.22) \times 10^{-3}$ & $(187.40 \pm 3.97) \times 10^{-3}$ & $7.26 \pm 0.19$ & $9.19 \pm 0.32$ \\
\hline 298 & $(15.49 \pm 0.76) \times 10^{-3}$ & $(177.36 \pm 2.04) \times 10^{-3}$ & $2.49 \pm 0.08$ & $2.97 \pm 0.02$ \\
\hline \multicolumn{5}{|c|}{ n-hexane } \\
\hline 313 & $(12.08 \pm 0.69) \times 10^{-3}$ & $(43.24 \pm 0.29) \times 10^{-3}$ & $(27.18 \pm 0.40) \times 10^{-3}$ & $(293.20 \pm 0.39) \times 10^{-6}$ \\
\hline 310 & $(9.69 \pm 0.75) \times 10^{-3}$ & $(29.63 \pm 0.25) \times 10^{-3}$ & $(25.88 \pm 0.90) \times 10^{-3}$ & $(184.34 \pm 0.57) \times 10^{-6}$ \\
\hline 298 & $(3.93 \pm 0.32) \times 10^{-3}$ & $(17.46 \pm 0.93) \times 10^{-3}$ & $(18.88 \pm 0.89) \times 10^{-3}$ & $(128.88 \pm 1.73) \times 10^{-6}$ \\
\hline
\end{tabular}


Table 1(a) - Solubility ranking at $298 \mathrm{~K}$ and respective binary systems

\begin{tabular}{ccc}
\hline Rank & $\begin{array}{c}\text { solubility range } \\
(\mathrm{g} / \mathrm{L})\end{array}$ & binary systems \\
\hline 7 & $>100$ & paracetamol/ethanol \\
\hline 6 & $10-100$ & paracetamol/water; paracetamol/acetone; budesonide/ethanol \\
\hline 5 & $1-10$ & $\begin{array}{c}\text { budesonide/acetone; budesonide/ethyl acetate; } \\
\text { furosemide/acetone; furosemide/ethanol; } \\
\text { furosemide/ethyl acetate }\end{array}$ \\
\hline 4 & $0.1-1$ & allopurinol/water; allopurinol/acetone; allopurinol/ethyl acetate; \\
budesonide/carbon tetrachloride
\end{tabular}


Table 2 - Average melting point and enthalpy of fusion of the studied drugs.

\begin{tabular}{|c|c|c|c|c|c|}
\hline \multirow{2}{*}{ Drugs } & \multirow{2}{*}{ Scans } & \multicolumn{2}{|c|}{$T_{m}(\mathrm{~K})$} & \multicolumn{2}{|c|}{$\Delta_{f u s} H\left(\mathrm{~kJ} \cdot \mathrm{mol}^{-1}\right)$} \\
\hline & & Exp. & $\begin{array}{c}\text { Group-contribution } \\
\text { method }^{\mathrm{a}}\end{array}$ & Exp. & $\begin{array}{c}\text { Group-contribution } \\
\text { method }^{\mathrm{a}}\end{array}$ \\
\hline paracetamol & 5 & $443.2 \pm 0.5$ & 385.5 & $27.6 \pm 1.1$ & 23.6 \\
\hline budesonide & 3 & $534.0 \pm 1.2$ & 477.8 & $34.7 \pm 1.2$ & 57.0 \\
\hline allopurinol & - & $653.5 \pm 1.3$ & 435.1 & ----- & 38.5 \\
\hline furosemide & - & $534.3 \pm 0.9$ & 510.9 & $\begin{array}{l}---- \\
--1\end{array}$ & 48.7 \\
\hline
\end{tabular}


Table 3(a) - NRTL-SAC molecular parameters $\left(r_{m, I}\right)$ for the solvents (Chen and Crafts, 2006).

\begin{tabular}{ccccc}
\hline Solvent & $\mathbf{X}$ & $\mathbf{Y}-$ & $\mathbf{Y +}$ & $\mathbf{Z}$ \\
\hline acetone & 0.131 & 0.109 & 0.513 & 0.000 \\
carbon tetrachloride & 0.739 & 0.027 & 0.142 & 0.000 \\
ethanol & 0.251 & 0.030 & 0.000 & 0.630 \\
ethyl acetate & 0.339 & 0.058 & 0.441 & 0.000 \\
n-hexane & 1.000 & 0.000 & 0.000 & 0.000 \\
water & 0.000 & 0.000 & 0.000 & 1.000 \\
\hline
\end{tabular}


Table 3(b) - NRTL binary parameters for conceptual segments in NRTL-SAC (Chen and Song, 2004).

\begin{tabular}{llllll}
\hline Segment 1 & $X$ & $X$ & $Y-$ & $Y+$ & $X$ \\
Segment 2 & $Y-$ & $Z$ & $Z$ & $Z$ & $Y+$ \\
\hline$\tau_{12}$ & 1.643 & 6.547 & -2.000 & 2.000 & 1.643 \\
$\tau_{21}$ & 1.834 & 10.949 & 1.787 & 1.787 & 1.834 \\
$\alpha_{12}=\alpha_{21}$ & 0.2 & 0.2 & 0.3 & 0.3 & 0.2 \\
\hline
\end{tabular}


Table 4 - Thermodynamic properties of dissolution of the drugs under study in pure solvents: $\Delta_{s o l} G(\mathrm{~kJ} / \mathrm{mol}), \Delta_{\text {sol }} H(\mathrm{~kJ} / \mathrm{mol})$ and $\Delta_{\text {sol }} S(\mathrm{~J} / \mathrm{mol} \cdot \mathrm{K})$.

\begin{tabular}{|c|c|c|c|c|c|c|c|c|c|c|c|c|}
\hline \multirow{2}{*}{$\begin{array}{c}\mathbf{T} \\
(\mathbf{K})\end{array}$} & \multicolumn{3}{|c|}{ paracetamol } & \multicolumn{3}{|c|}{ allopurinol } & \multicolumn{3}{|c|}{ budesonide } & \multicolumn{3}{|c|}{ furosemide } \\
\hline & $\Delta_{\text {sol }} \mathbf{H}$ & $\Delta_{\text {sol }} \mathbf{G}$ & $\Delta_{\text {sol }} S$ & $\Delta_{\text {sol }} H$ & $\Delta_{\text {sol }} \mathbf{G}$ & $\Delta_{\text {sol }} S$ & $\Delta_{\text {sol }} H$ & $\Delta_{\text {sol }} \mathbf{G}$ & $\Delta_{\text {sol }} S$ & $\Delta_{\text {sol }} H$ & $\Delta_{\text {sol }} \mathbf{G}$ & $\Delta_{\text {sol }} S$ \\
\hline \multicolumn{13}{|c|}{ water } \\
\hline 315 & 38.48 & 14.14 & 77.23 & 53.67 & 24.77 & 91.71 & 14.78 & 35.98 & -67.27 & 35.34 & 33.28 & 6.55 \\
\hline 313 & 37.99 & 14.44 & 75.22 & 52.99 & 24.77 & 90.14 & 14.59 & 35.89 & -68.01 & 34.89 & 33.57 & 4.22 \\
\hline 310 & 37.27 & 14.74 & 72.63 & 51.98 & 25.23 & 86.25 & 14.32 & 35.59 & -68.59 & 34.23 & 33.92 & 0.99 \\
\hline 303 & 35.61 & 15.12 & 67.59 & 49.66 & 25.66 & 79.19 & 13.68 & 35.15 & -70.84 & 32.70 & 33.56 & -2.83 \\
\hline 298 & 34.44 & 15.41 & 63.82 & 48.04 & 26.15 & 73.43 & 13.23 & 34.80 & -72.36 & 31.63 & 33.44 & -6.06 \\
\hline \multicolumn{13}{|c|}{ acetone } \\
\hline 313 & 18.29 & 7.17 & 35.52 & 59.76 & 17.22 & 135.85 & 40.11 & 17.03 & 73.70 & 33.10 & 18.07 & 48.00 \\
\hline 310 & 17.94 & 7.25 & 34.46 & 58.62 & 18.66 & 128.84 & 39.35 & 17.37 & 70.86 & 32.47 & 18.84 & 43.96 \\
\hline 298 & 16.58 & 7.65 & 29.94 & 54.17 & 19.70 & 115.63 & 36.36 & 18.08 & 61.30 & 30.01 & 18.91 & 37.21 \\
\hline \multicolumn{13}{|c|}{ carbon tetrachloride } \\
\hline 313 & 85.20 & 29.08 & 179.21 & 20.50 & 24.82 & -13.79 & 34.16 & 22.39 & 37.59 & 90.25 & 37.98 & 166.93 \\
\hline 310 & 83.57 & 29.51 & 174.32 & 20.11 & 25.11 & -16.14 & 33.51 & 22.92 & 34.15 & 88.53 & 38.98 & 159.76 \\
\hline 298 & 77.23 & 31.54 & 153.25 & 18.58 & 24.67 & -20.43 & 30.97 & 23.01 & 26.69 & 81.81 & 40.44 & 138.77 \\
\hline \multicolumn{13}{|c|}{ ethanol } \\
\hline 313 & 12.31 & 6.40 & 18.89 & 74.19 & 25.08 & 156.82 & 37.91 & 14.23 & 75.63 & 97.84 & 15.28 & 263.62 \\
\hline 310 & 12.08 & 6.52 & 17.91 & 72.78 & 26.14 & 150.36 & 37.19 & 14.78 & 72.25 & 95.97 & 17.32 & 253.59 \\
\hline 298 & 11.16 & 6.67 & 15.05 & 67.25 & 27.45 & 133.49 & 34.37 & 15.38 & 63.69 & 88.69 & 19.42 & 232.34 \\
\hline \multicolumn{13}{|c|}{ ethyl acetate } \\
\hline 313 & 36.20 & 28.08 & 25.93 & 7.66 & 22.89 & -48.63 & 67.75 & 16.30 & 164.29 & 71.58 & 14.99 & 180.73 \\
\hline 310 & 35.51 & 28.58 & 22.34 & 7.52 & 22.97 & -49.83 & 66.46 & 16.51 & 161.03 & 70.22 & 15.23 & 177.30 \\
\hline 298 & 32.81 & 28.52 & 14.39 & 6.95 & 22.22 & -51.22 & 61.42 & 18.53 & 143.86 & 64.89 & 17.44 & 159.15 \\
\hline \multicolumn{13}{|c|}{ n-hexane } \\
\hline 313 & 61.07 & 29.84 & 99.70 & 45.49 & 26.25 & 61.45 & 20.30 & 30.46 & -32.43 & 38.97 & 41.56 & -8.28 \\
\hline 310 & 59.90 & 30.12 & 96.01 & 44.63 & 26.97 & 56.92 & 19.91 & 30.29 & -33.46 & 38.23 & 42.36 & -13.31 \\
\hline 298 & 55.36 & 31.20 & 81.03 & 41.24 & 27.24 & 46.95 & 18.40 & 29.90 & -38.56 & 35.33 & 41.61 & -21.08 \\
\hline
\end{tabular}


Table 5 - Regressed NRTL-SAC molecular parameters $\left(r_{m, I}\right)$ for the studied compounds in all binary systems except water and respective absolute average deviation (AAD).

\begin{tabular}{cccccc}
\hline Solute name & $\mathbf{X}$ & $\mathbf{Y}-$ & $\mathbf{Y +}$ & $\mathbf{Z}$ & $\mathbf{A A D}(\boldsymbol{\%})^{\mathbf{a}}$ \\
\hline paracetamol & 0.416 & 0.016 & 0.168 & 1.861 & 65 \\
budesonide & 1.000 & 0.178 & 0.005 & 1.079 & 82 \\
allopurinol & 0.016 & 0.002 & 1.169 & 0.000 & 39 \\
furosemide & 0.600 & 0.127 & 0.010 & 1.620 & 83 \\
\hline
\end{tabular}

${ }^{\mathrm{a}} A A D(\%)=\frac{1}{N} \sum_{i}\left(\frac{x_{s}^{\exp }-x_{s}^{\text {calc }}}{x_{s}^{\exp }} \times 100\right)$ 


\section{Figure Captions:}

Figure 1 - Chemical structures of the drugs under study.

Figure 2 - Solubility of paracetamol in several solvents: this work vs. literature water [ (Bustamante et al., 1998a) at 298 and 303 K, - (Granberg and Rasmuson, 1999) at 298, 303 and $313 \mathrm{~K}, \boldsymbol{\Delta}$ (Hojjati and Rohani, 2006b) at 298, 303 and $313 \mathrm{~K}$ ], acetone [x (Granberg and Rasmuson, 2000) at $298 \mathrm{~K}$, carbon tetrachloride [(* (Granberg and Rasmuson, 1999) at $298 \mathrm{~K}$ ], ethanol [• (Granberg and Rasmuson, 1999) at $298 \mathrm{~K}$, (Jimenez and Martinez, 2006) at 298 and $303 \mathrm{~K}, \diamond$ (Romero et al., 1996) at 298, 303 and $313 \mathrm{~K}$ ], ethyl acetate [ ${ }^{\circ}$ (Granberg and Rasmuson, 1999) at $303 \mathrm{~K}$ ], n-hexane [ $\square$ (Perlovich et al., 2006) at 298 and $310 \mathrm{~K}]$.

Figure 3 - NRTL-SAC prediction results: $\bullet$, paracetamol; $\boldsymbol{\Delta}$, budesonide; $\square$, furosemide; $\oplus$, allopurinol.

Figure 4 - NRTL-SAC prediction results for paracetamol solubility in ethanol-ethyl acetate mixed solvent at 298.2 K ( $\diamond$, Romero et al. (1996); - NRTL-SAC predictions) with the parameters estimated in this work; --, NRTL-SAC predictions) with the parameters obtained by Chen and Crafts (2006). 
Figure 1:

Paracetamol<smiles>CC(=O)Nc1ccc(O)cc1</smiles>

\section{Budesonide}

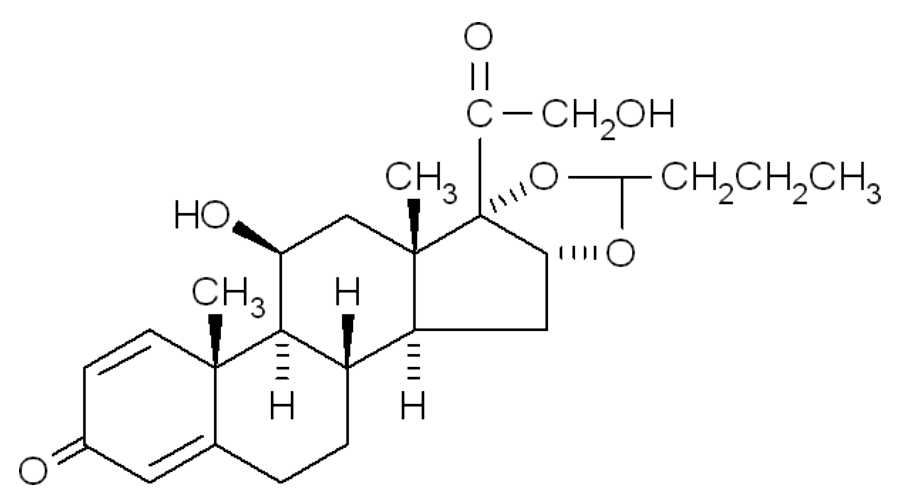

Furosemide<smiles>NS(=O)(=O)c1cc(C(=O)O)c(NCc2ccco2)cc1Cl</smiles>

Allopurinol

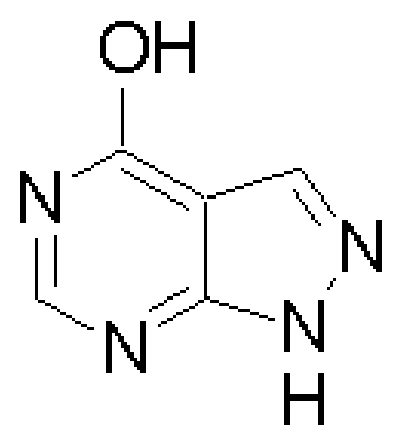


Figure 2:

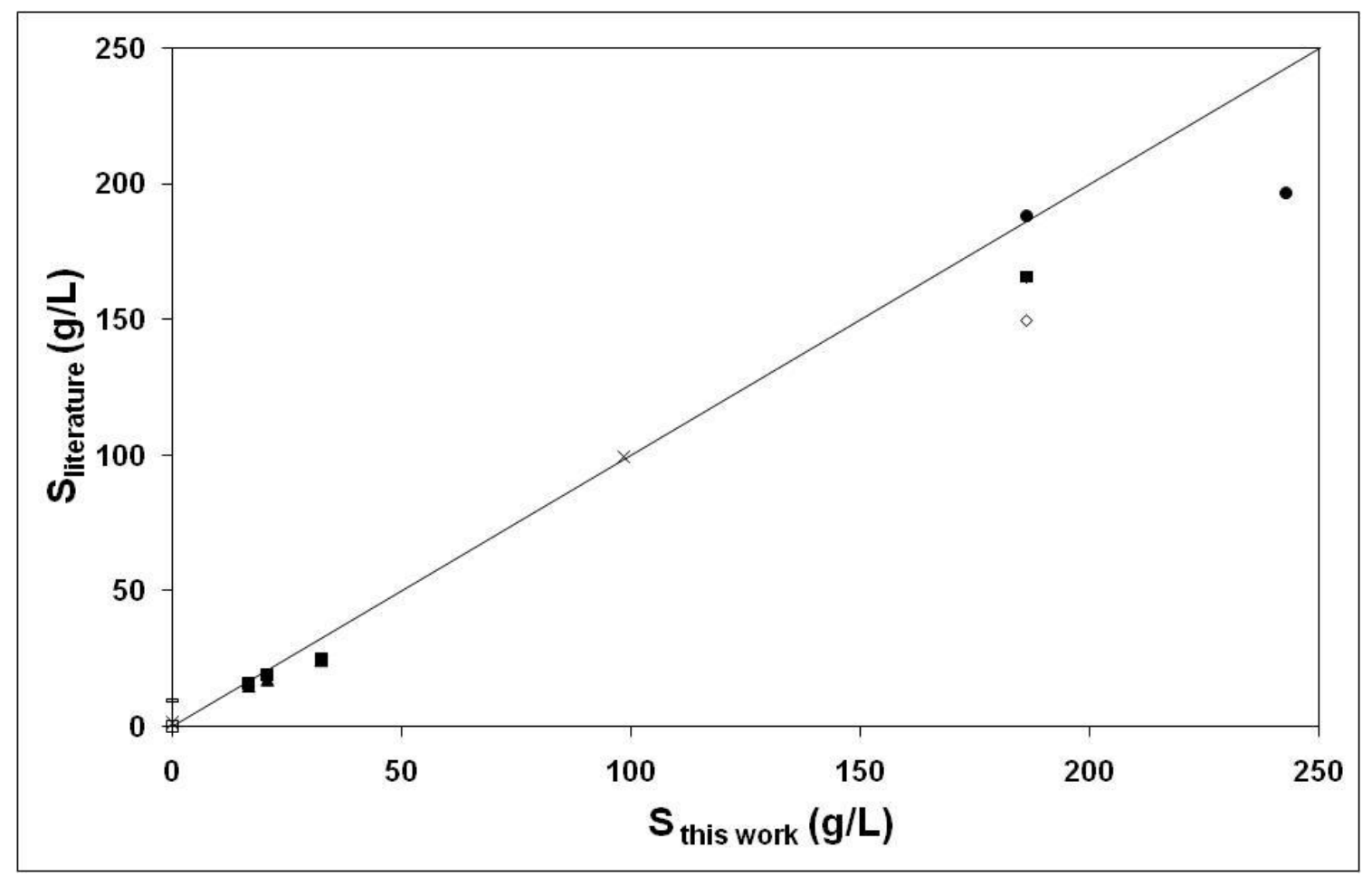


Figure 3:

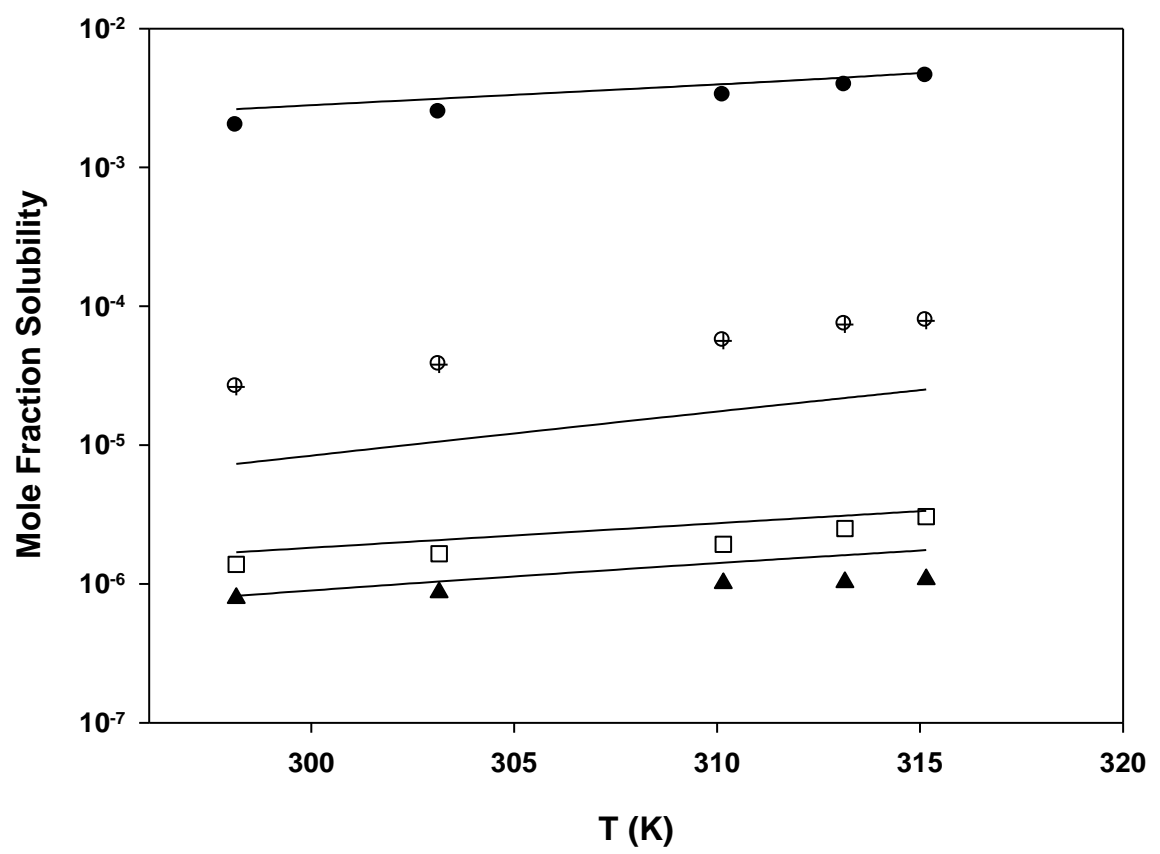


Figure 4:

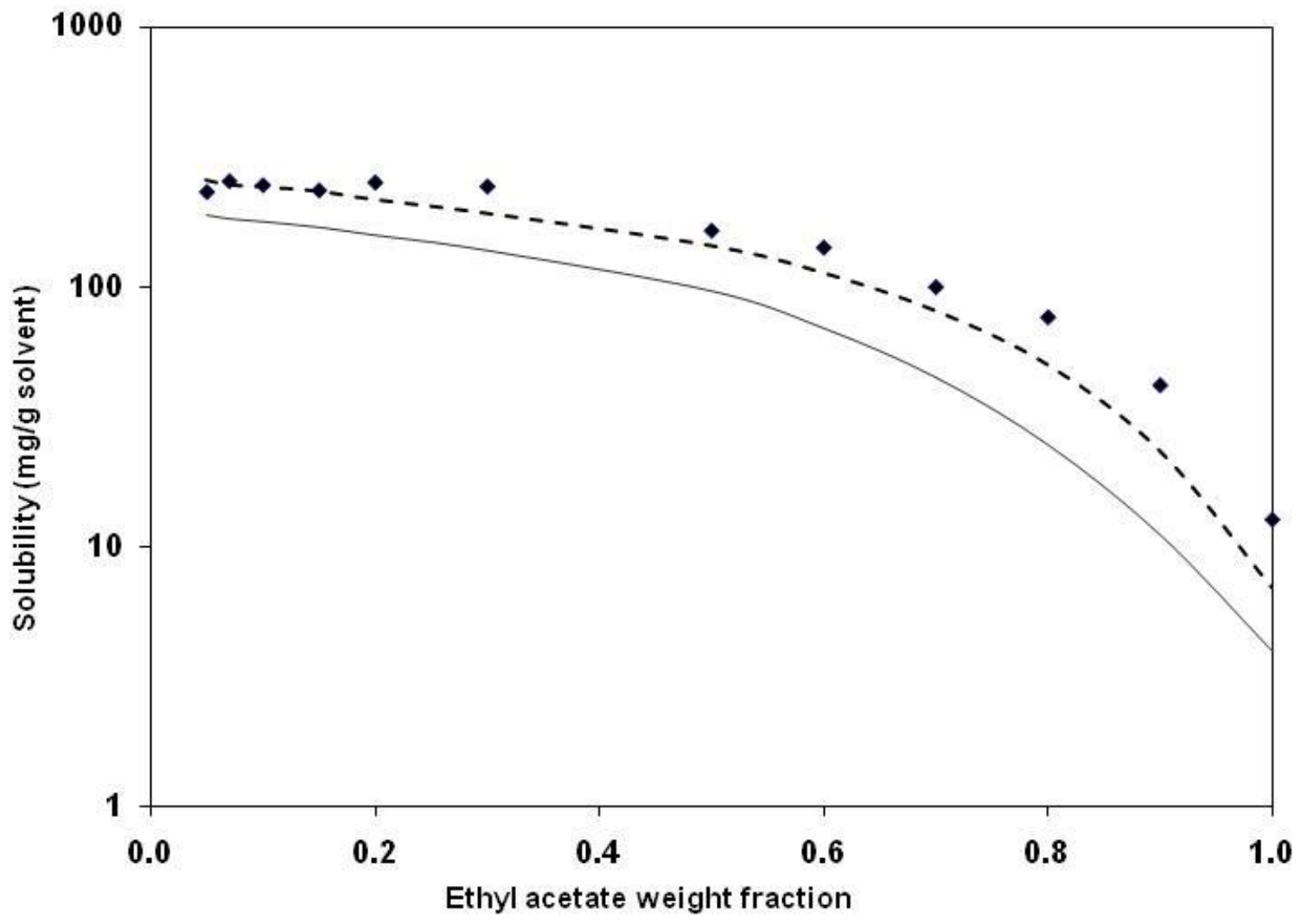

